\title{
Bordetella holmesii: Comparison of Two Isolates from Blood and a Respiratory Sample
}

\author{
Valérie Bouchez ${ }^{1,2}$, Nicole Guiso ${ }^{1,2}$ \\ ${ }^{1}$ Institut Pasteur, Unité Prévention et Thérapie Moléculaires des Maladies Humaines, Paris, France; ${ }^{2}$ Centre National de la Recherche \\ Scientifique (CNRS), Paris, France. \\ Email: valerie.bouchez@pasteur.fr
}

Received February $14^{\text {th }}, 2013$; revised March $28^{\text {th }}, 2013$; accepted April $28^{\text {th }}, 2013$

Copyright (C) 2013 Valérie Bouchez, Nicole Guiso. This is an open access article distributed under the Creative Commons Attribution License, which permits unrestricted use, distribution, and reproduction in any medium, provided the original work is properly cited.

\begin{abstract}
Interest in Bordetella holmesii is increasing, but very little is known about this bacterium, which can be isolated from both blood and respiratory samples. In this study, we compared a B. holmesii isolate from the blood sample of an adult with bacteremia with another isolate from a nasopharyngeal swab from an adult with whooping cough syndrome. Genetic analysis was carried out, targeting relevant genes, and virulence properties were studied in cellular and animal models. Our genomic analysis provided no evidence of traits specific to either blood or respiratory isolates of B. holmesii. Neither isolate was cytotoxic to human tracheal epithelial cells. Both isolates were only weakly invasive and they did not persist within epithelial cells for less than $48 \mathrm{~h}$.
\end{abstract}

Keywords: Bordetella holmesii; Bacteremia; Respiratory Isolate

\section{Introduction}

Bordetella holmesii is a Gram-negative bacterium, first described in 1995, following its isolation from the blood of a patient with septicemia [1]. Phylogeny analysis based on 16S rRNA genes initially placed $B$. holmesii close to $B$. pertussis. However, multilocus sequence typing showed that $B$. holmesii did not belong to the classical Bordetella group (B. pertussis, B. parapertussis and $B$. bronchiseptica), being instead more closely related to $B$. avium [2]. Very few putative virulence factors have been identified in this species: a two-component bvg operon (bvgA and $b v g S$ ) [3,4], a filamentous hemagglutinin-like protein FHA-bho [5] and an alcaligin operon [2]. By contrast, no toxin or adhesion similar to those involved in pathogenesis in classical Bordetella species has been identified. There has recently been an increase in the number of cases linking $B$. holmesii to whooping cough syndromes. The $B$. holmesii genome harbors insertion sequences, such as IS481, which is also present in the genome of $B$. pertussis, but at a lower copy number [6]. This poses a potential problem, because the diagnosis of $B$. pertussis infection is based on the PCR detection of IS481 sequences. The development of specific PCR diagnosis for $B$. holmesii infection, targeting the rec $A$ gene [7-9], or the transposase IS1001bho [10], the amplifica- tion of specific target as bhoE [11] or the sequencing of 16sRNA or OmpA [12] has made it possible for a number of retrospective studies to demonstrate an increase in the number of reported cases of respiratory infections due to $B$. holmesii over the last few years [10,11,13-21]. We wondered whether this increase in detection reflected a real increase in the number of infections or was simply a consequence of the increasing use of RT-PCR targeting IS481 for the diagnosis of $B$. pertussis since 2005. Indeed, Njamkepo et al. [16] reported a prevalence of 6.8\% for $B$. holmesii in respiratory samples from patients with a principal diagnosis of pertussis. Prevalence was highest (20.3\%) in adolescents and adults, and no cases were found in children under the age of nine years. By contrast, 1) during an outbreak in Chile, Miranda et al. [22] reported a prevalence of $11.1 \%$ for $B$. holmesii, mostly in adolescents and young adults, but they also identified three cases (3\%) in patients under the age of one year; 2) in Argentina in 2010, Bottero et al., [21] reported 9 cases due to $B$. holmesii, among 1475 pertussis suspected cases patients, 7 under 6 months of age, one 7 months old and one 13 years old. Rodgers et al. [10] recently reported that, during an outbreak of pertussis in Ohio (USA), 30\% of the patients were infected with B. holmesii and not with $B$. pertussis. These findings raise questions concerning the nature of $B$. holmesii: is it an op- 
portunistic bacterium or an invasive or respiratory pathogen? They also raise questions about the possible differences between invasive and respiratory isolates. The aim of our study was to compare two B. holmesii isolates, one from blood [23] and the other from a respiratory sample [6], in terms of their genetics, targeting relevant genes and considering their virulence in cellular and animal tests.

\section{Materials and Methods}

\subsection{Bacterial Growth and DNA Extraction}

All B. pertussis and B. holmesii isolates used were obtained from the Collection of the Pasteur Institute or the French National Reference Center. They were grown at $36^{\circ} \mathrm{C}$ for 72 hours on Bordet-Gengou agar (BGA) supplemented with $15 \%$ defibrinated sheep blood, and replated on the same medium and incubated for 24 hours before use. A list of the isolates used is presented in Table 1. For pyrosequencing, genomic DNA was prepared on Genomic-tip 500/G anion-exchange columns (Qiagen), according to the manufacturer's recommendations. For PCR validation, DNA extractions were performed with the DNeasy Blood \& Tissue Kit (Qiagen) according to the manufacturer's instructions.

\subsection{Pulsed-Field Gel Electrophoresis}

DNA fingerprinting was performed by pulsed-field gel electrophoresis (PFGE), as previously described [24].

\subsection{Western-Blot Analysis}

Western blots were carried out as described by Weber et al. [25].

\subsection{Pyrosequencing and In-House Sequence Verifications}

Pyrosequencing was carried out with a 454GS-FLX NextGen sequencing platform (Roche Diagnostics GmbH, Beckman Coulter Genomics). Three mate-pair libraries were constructed for both isolates. The two samples were then simultaneously sequenced in one GS-FLX run, with a $70 \times 75 \mathrm{~mm}$ Pico-Titer plate device (Roche Diagnostics $\mathrm{GmbH}$ ) and the GS LR-70 sequencing kit (Roche Diagnostics $\mathrm{GmbH}$ ), as previously described [26]. A standard sequencing assembly was generated with Newbler assembler version 2.3 from 454/Roche, generating 11 scaffolds for Bho1 and 33 for FR4020. However, as no reference annotated genome is available for $B$. holmesii, we initially focused on the few genes of interest already identified for this species: $b v g A / S$ [3], fhaB [5], the alcaligin operon and $B$. holmesii-specific genes [2]. Moreover, as pyrosequencing technology is known to generate errors, particularly in homopolymeric nucleotide tracts, we checked all the sequence differences ob- served in these genes by PCR and classical Sanger sequencing for both the isolates studied and for some additional isolates (Table 1). The primers used are listed in Table 2. Concatenated sequences of the four sequences of interest (located in $b v g A / b v g S /$ fhaB and fimC) were aligned for the construction of a neighbor-joining tree, with PHYLIP software [27] from the Pasteur Mobyle Portal (Figure 1).

We then used the NCBI blastn and blastp programs to identify other genes potentially important for the putative pathogenicity of $B$. holmesii.

\subsection{Cell Culture}

The murine monocyte/macrophage-like cell line J774.A1 and human tracheal epithelial cells (HTE) were cultured as previously described [26]

\subsection{Cytotoxicity Assays}

Bacterial cytotoxicity to J774.A1 and HTE cells was assessed as previously described [26]. Briefly, bacteria were added to cells at a ratio of 100 bacteria per cell and gently centrifugated. Infected cells were incubated at $37^{\circ} \mathrm{C}$, in the presence of $5 \% \mathrm{CO}_{2}$, for 8 hours. Cytotoxicity was determined every 2 hours, with the Cytotox $96 \circledR$ Non-Radioactive Cytotoxicity Assay (Promega), which measures the activity of the lactate dehydrogenase released into the culture supernatant. In each experiment with J774.A1, we used Tohama, the B. pertussis reference strain known to be cytotoxic to these cells, as a positive control [28]. In each experiment with HTE cells, we used RB50, the $B$. bronchiseptica reference strain known to be cytotoxic to these cells, as a positive control [29].

\subsection{HTE Cell Invasion and Persistence Assays}

Invasion and persistence assays were conducted with HTE cells, as previously described [26]. Briefly, bacteria were added to cells at a ratio of 100 bacteria per cell and gently centrifugated. Infected cells were incubated at $37^{\circ} \mathrm{C}$, in the presence of $5 \% \mathrm{CO}_{2}$. After 5 hours of incubation, cells were thoroughly washed and incubated for a further two hours with gentamicin (Sigma) at a final concentration of $100 \mu \mathrm{g} / \mathrm{ml}$, to kill any remaining extracellular bacteria. For persistence assays, the concentration of gentamicin in the cell culture medium was then decreased to $10 \mu \mathrm{g} / \mathrm{ml}$ over the rest of the incubation period. The number of intracellular Bordetella was determined by cell lysis and determinations of the number of CFU after 5 hours for invasion assays and after 48 hours of incubation for persistence assays.

The results presented are the means and standard deviations of at least three experiments. The symbol $\left(^{*}\right)$ in Figure 2 indicates $p<0.05$ in comparisons with the re- 
Table 1. List of clinical isolates used in the study.

\begin{tabular}{|c|c|c|c|c|c|c|}
\hline Name of isolate & Species & Year of collection & Origin & Age of the patient & Disease/pathological detection & Reference \\
\hline CIP104394 (ATCC51541-F5101) & $\mathrm{Bh}^{*}$ & 1983 & USA & 37 & Mild leukocytosis, cardiomegaly & [1] \\
\hline CIP104395 (G7702) & $\mathrm{Bh}$ & 1992 & USA & 36 & Dog bite & [1] \\
\hline CIP104396 & $\mathrm{Bh}$ & $\mathrm{Nk}^{\S}$ & Saudi Arabia & 32 & Endocarditis & [1] \\
\hline Bho1 & $\mathrm{Bh}$ & 1996 & France & 20 & Hemoculture/asplenic & [23] \\
\hline FR3713 & $\mathrm{Bp}^{* *}$ & 2007 & France & 0.17 & Respiratory & [6] \\
\hline FR3791 & $\mathrm{Bh}$ & 2007 & France & 20 & Hemoculture/asplenic & [6] \\
\hline FR4020 & $\mathrm{Bh}$ & 2008 & France & 44 & Respiratory & [6] \\
\hline CA001 & $\mathrm{Bh}$ & before 2008 & Canada & 13 & Hemoculture/asplenic & [20] \\
\hline CA002 & $\mathrm{Bh}$ & before 2008 & Canada & 17 & Hemoculture/asplenic & [20] \\
\hline CA003 & $\mathrm{Bh}$ & before 2008 & Canada & 13 & Hemoculture/asplenic & [20] \\
\hline CA004 & $\mathrm{Bh}$ & before 2008 & Canada & 15 & Hemoculture/asplenic & [20] \\
\hline FR4060 & $\mathrm{Bh}$ & 2009 & France & 10 & Hemoculture & [6] \\
\hline FR4101 & $\mathrm{Bh}$ & 2009 & France & 15 & Respiratory & [6] \\
\hline FR4645 & $\mathrm{Bh}$ & 2009 & France & 15 & Respiratory & [6] \\
\hline FR4859 & $\mathrm{Bh}$ & 2010 & France & 67 & Hemoculture & [6] \\
\hline FR4884 & $\mathrm{Bh}$ & 2011 & France & 68 & Respiratory & [6] \\
\hline FR4912 & $\mathrm{Bh}$ & 2011 & France & 61 & Respiratory & [6] \\
\hline
\end{tabular}

$\mathrm{Nk}^{\S}$ : Not known; $\mathrm{Bh}^{*}:$ Bordetella holmesii; $\mathrm{Bp}^{* *}:$ Bordetella pertussis.

Table 2. List of primers used in the study.

\begin{tabular}{|c|c|c|c|}
\hline Target gene & Primer name & Primer sequence (5'->3') & PCR product size (bp) \\
\hline \multirow{2}{*}{ bvgA } & bvgA-bho-F & AAAGAGGGTTTCGAGGTCGT & \multirow{2}{*}{543} \\
\hline & $b v g A-b h o-R$ & TCGTTTGGCCATGTCTATCA & \\
\hline \multirow{4}{*}{ bvgS } & bvgS1-bho-F & AGTCACAACCTGGGCTTGAT & \multirow{2}{*}{760} \\
\hline & bvgS1-bho-R & CGATCTGGAAATGCAATCCT & \\
\hline & bvgS2-bho-F & CGCAACAATTGGCATACATC & \multirow{2}{*}{611} \\
\hline & bvgS2-bho-R & CATATCGCCCTTGCTTTGAC & \\
\hline \multirow{12}{*}{ fhaB-bho } & fhaB1_bho-F & GGTTTGCAGACCTTCGACAT & \multirow{2}{*}{587} \\
\hline & fhaB1-bho-R & ATGTCACGCGACAGGATGAT & \\
\hline & fhaB2-bho-F & GGCACGCAGGTCTTGATG & \multirow{2}{*}{808} \\
\hline & fhaB2-bho-R & CCGTGCTGCTGAAGTTGG & \\
\hline & fhaB3-bho-F & GACCATCAAGACCGAGCAGT & \multirow{2}{*}{656} \\
\hline & fhaB3-bho-R & GTACGAAGTTTGCGTGTGGA & \\
\hline & fhaB4-bho-F & CCGGTATCCGAACTCAAGAC & \multirow{2}{*}{301} \\
\hline & fhaB4-bho-R & CGTTCTGCTTGCTGTTGTTG & \\
\hline & fhaB5-bho-F & GCAAGATCCAGGCTGACAAC & \multirow{2}{*}{316} \\
\hline & fhaB5-bho-R & GCGATCTGGCTGTAGTTGGT & \\
\hline & fhaB6-bho-F & TTCCAGAAACTGGGCTATCAA & \multirow{2}{*}{273} \\
\hline & fhaB6-bho-R & GTTTATCCGCGTCCAGACAT & \\
\hline Upstream & pfhaBbho-F & GGCCTGTTGCATGAGTCTCT & \multirow{2}{*}{368} \\
\hline fhaB & pfhaBbho-R & GGCGTTCACGGTGTATCTCT & \\
\hline \multirow{2}{*}{ fimC } & fimCbho-F & TTCTACGGGACGAGCTATCG & \multirow{2}{*}{307} \\
\hline & fimCbho-R & AAACGCСТАССССТTTGACT & \\
\hline
\end{tabular}




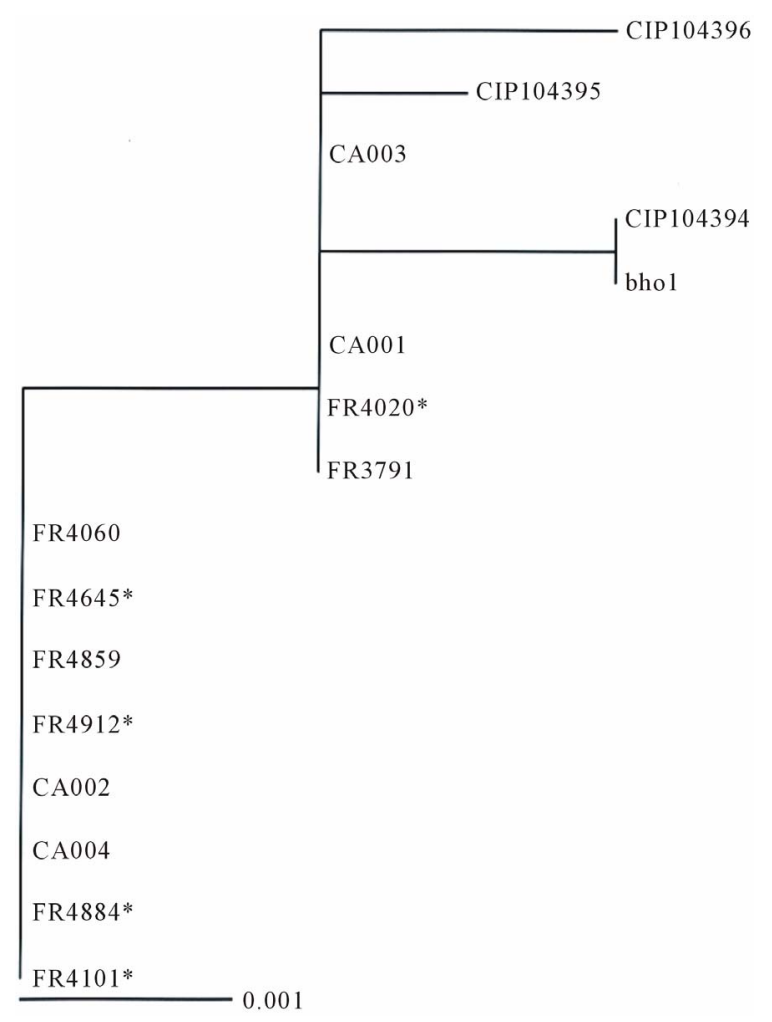

Four concatenated sequences of interest (located in $b v g A / b v g S / f h a B$ and $\operatorname{fimC}$ ) were aligned for the construction of a neighbor-joining tree. The symbol $\left({ }^{*}\right)$ is used to identify isolates of respiratory origin.

Figure 1. Neighbor-joining tree based on partial sequences

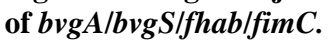

cently isolated B. pertussis FR3713.

\subsection{Animal Models}

All procedures involving animals were conducted in accordance with the Institut Pasteur animal care and use committee guidelines. $\mathrm{LD}_{50}$ was determined as previously described [26].

\section{Results}

\subsection{Description of the Isolates}

Table 1 presents the isolates used in this study. We compared one isolate from a man with sickle cell anemia and B. holmesii bacteremia (Bho1, [23]) with another isolate from the respiratory tract of a woman with whooping cough syndrome (FR4020, [6]). These two isolates had PFGE profiles (data not shown) identical to those of the other isolates tested (Table 1) $[6,20,23]$. None of the toxins and adhesins involved in the pathogenicity of classical Bordetella species, such as pertussis toxin (PT), adenylate cyclase-hemolysin toxin (ACHly), Bordetella type 3 secretion system effector A (BteA), filamentous hemagglutinin (FHA), pertactin (PRN) and fimbrial proteins (Fim 2 and Fim 3), were detected in suspensions of the isolates with specific antibodies raised against purified $B$. pertussis virulence factors.

\subsection{Pyrosequencing and In-House Sequence Verification}

We used pyrosequencing data and carried out in-house PCR sequence verifications, focusing on the small number of genes already identified as of potential interest for studies of the physiopathology of this species.

\subsubsection{The bvgAS Regulatory System}

We compared the sequences obtained for $b v g A$ and $b v g S$ between Bho1 and FR4020. They were found to display 99.9\% and 99.8\% nucleotide identity, respectively. We also compared these sequences with the sequences deposited in GenBank for the B. holmesii G7702 isolate (AJ748854.1) [3] and with the corresponding sequences obtained from additional isolates.

Differences in the bvgS sequence were found in positions $1973(\mathrm{G} / / \mathrm{T}), 2036(\mathrm{~A} / / \mathrm{G}), 2191(\mathrm{G} / / \mathrm{T}), 2252(\mathrm{~A} / / \mathrm{G})$, 4894 (G//A) and 4889 (T//G) (Table 3). All but one of these SNPs resulted in an amino-acid substitution, the remaining SNP, that in position 2252, being silent. Three SNPs (in positions 2252, 4894 and 4889) were found in all the isolates tested, regardless of the sequencing technique used. These SNPs were also found in CIP104395 (derived from the G7702 isolate used for the bvgS GenBank sequence determination), suggesting a possible error in the sequence present in GenBank at these positions. CIP104396 was the only isolate with a G in position 2036 and Bho1 and CIP101394 were the only isolates with a T rather than a G in positions 1973 and 2191. In conclusion, with the exception of CIP104396, Bho1 and CIP104394, the respiratory and bacteremia-related isolates tested had identical bvgS sequences.

Differences were also observed in $b v g A$, at positions 1085 (additional A), 1220 (G//A) and 1403 (A//G) (Table 3). All isolates except CIP104395 (derived from G7702) displayed a silent SNP (A//G) at position 1403. CIP104396 was the only isolate with an A in position 1220, whereas both the Bho1 and CIP104394 isolates had an additional A residue in position 1085, leading to premature termination of the activator $b v g A$.

\subsubsection{The FHA-Related Protein}

We observed a sequence with some similarities to that of fhaB, as reported by Link et al. [5], in the genomes of Bho1 and FR4020. This sequence displayed 99.98\% nucleotide sequence identity between these two isolates. We also compared these sequences with the sequences for $B$. holmesii obtained with the G7702 isolate and deposited in GenBank (AM491633.1) [5] and with the corresponding sequences obtained from additional isolates. Differences were observed in positions $151(\mathrm{C} / / \mathrm{T}), 3094$ 
Table 3. Sequence differences observed in B. holmesii isolates for $b v g S, \operatorname{bvgA}$, fhaB-bho and fimC.

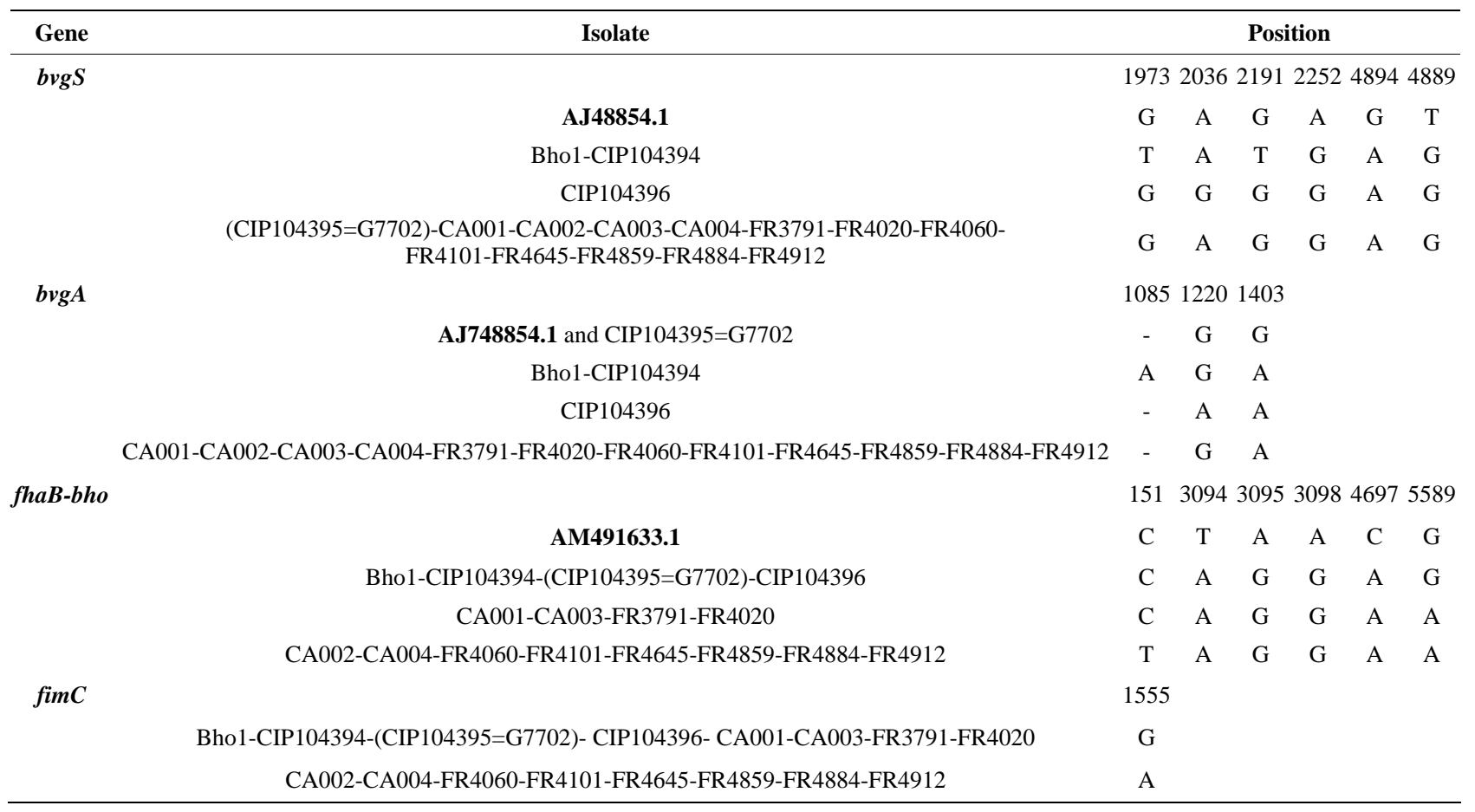

(T//A), 3095 (A//G), 3098 (A//G), 4697 (C//A) and 5589 (G//A) (Table 3). Four of these SNPs (position 3094, 3095, 3098 and 4697) were found in all the isolates tested, including CIP104395 (derived from G7702), regardless of the sequencing technique used, suggesting a possible error in the GenBank sequence at these positions. Bho1, CIP104394, CIP104395 and CIP104396 displayed only these four differences. An additional silent mutation was found at position 5589 in all the other isolates and a SNP at position 151 was found only in the most recent isolates, whether obtained from blood or respiratory system samples. This SNP results in a $\mathrm{Y}_{51}$ residue, rather than the $L_{51}$ present in the signal domain of the protein.

\subsubsection{Other Putative Virulence Related CDS}

CDS analogous to fimABCD-fhaC were detected, with a $\%$ GC of up to $62 \%$ (GenBank accession KC203605). The sequences for each CDS were identical in the two isolates tested, except for fimC which displayed one difference: Bho1 and FR4020 had a G in position 1555 of fimC, whereas the other isolates had an A. This difference results in a difference in the amino acid sequence of the corresponding protein, with an S residue replacing the original residue present at position 372 in fimC.

A CDS analogus to ampG involved in TCT [30] release has also been identified in both isolates.

\subsubsection{Neighbor-Joining Tree}

Concatenated sequences of the four sequences of in- terest (located in $b v g A / b v g S / f h a B$ and fimC) were aligned for the construction of a neighbor-joining tree (Figure 1). This tree showed that the $B$. holmesii isolates tested belonged to two main groups. The first consisted of FR4020, FR3791, CA001 and CA003 and three nodes corresponding to Bho1 and CIP104394 together, CIP104395 and CIP104396. The second group contained all the other isolates tested, corresponding to the most recent isolate, regardless of their blood or respiratory origin.

\subsubsection{Iron Uptake Island, Specific bho CDS and the Alcaligin Operon}

We confirmed the presence of the $B$. holmesii-specific bhoABCDE genes in all the isolates tested and found that the sequences of these genes were identical to those submitted to GenBank (DQ402419) [2]. The genes of the alcaligin operon, alcABCDERS-fauA, were conserved in both isolates, which displayed similar differences from those of B. pertussis (BP2456 to BP2463, see Table 4). PCR with previously described primers [2] targeting this region was carried out to check that the organization of this alcaligin operon was conserved.

\subsubsection{Diagnosis Targets}

Bho1 and FR4020 displayed no differences in recA, bhoE or ompA sequences.

\subsubsection{Putative Acquired CDS}

We identified two loci as potentially acquired from other 
Table 4. Sequence differences observed with $B$. pertussis sequences (NC_002929) for the alcaligin operon of Bho1 and FR4020.

\begin{tabular}{|c|c|c|c|c|c|c|c|c|}
\hline \multirow{2}{*}{$\begin{array}{l}\text { Gene } \\
\text { alcA }\end{array}$} & \multirow{2}{*}{$\begin{array}{c}\text { Ref. B. pertussis NC_002929 } \\
\text { BP2456 }\end{array}$} & \multicolumn{6}{|c|}{ Position } & \multirow[t]{2}{*}{ B. holmesii isolates } \\
\hline & & 732(T) & $788(\mathrm{~T})$ & $1088(C)$ & & & & \\
\hline & & $\mathrm{C}$ & $\mathrm{C}$ & $\mathrm{T}$ & & & & Bho1-FR4020 \\
\hline \multirow[t]{2}{*}{ alcB } & BP2457 & $45(\mathrm{G})$ & & & & & & \\
\hline & & A & & & & & & Bho1-FR4020 \\
\hline \multirow[t]{2}{*}{ alcC } & BP2458 & $145(\mathrm{~T})$ & $583(\mathrm{~T})$ & $645(G)$ & $858(C)$ & 891(C) & 1282(G) & \\
\hline & & $\mathrm{C}$ & $\mathrm{C}$ & A & $\mathrm{T}$ & $\mathrm{T}$ & A & Bho1-FR4020 \\
\hline \multirow[t]{2}{*}{ alcD } & BP2459 & $222(\mathrm{~T})$ & $474(\mathrm{C})$ & $571(\mathrm{G})$ & & & & \\
\hline & & $\mathrm{C}$ & $\mathrm{T}$ & A & & & & Bho1-FR4020 \\
\hline \multirow[t]{2}{*}{ alcR } & BP2461 & $6(\mathrm{~A})$ & 295(A) & & & & & \\
\hline & & G & G & & & & & Bho1-FR4020 \\
\hline \multirow[t]{2}{*}{ alcS } & ВР2463 & 938(C) & $972(G)$ & 1204(G) & & & & \\
\hline & & $\mathrm{T}$ & A & A & & & & Bho1-FR4020 \\
\hline \multirow[t]{2}{*}{ FauA } & BP2463 & 75(C) & 1101(C) & 1392(C) & 1603(A) & $1850(C)$ & & \\
\hline & & $\mathrm{T}$ & $\mathrm{T}$ & A & G & $\mathrm{T}$ & & Bho1-FR4020 \\
\hline
\end{tabular}

species (GenBank accession KC203606-KC203607): 1) the first had a mean \% GC of $57 \%$ and contained genes displaying sequence similarity to genes encoding a benzene monooxygenase, $b m o A$, and a toluene O-xylene monooxygenase subunit from Pseudomonas mendocina, 2) the second had a mean \% GC of $61 \%$ and contained genes displaying sequence similarity to hypothetical protein-encoding genes from Azoarcus aromaticum.

\subsection{LD50}

Whatever their origin, Bho1 and FR4020 were not lethal in a murine model of respiratory infection, even after inoculation with a dose of $2 \times 10^{8} \mathrm{CFU} / \mathrm{mouse}$.

\subsection{Interaction with Cells}

Contrary to B. pertussis, both B. holmesii isolates tested were not cytotoxic for murine macrophages cell line J774.A1, correlating with the absence of AC-Hly activity and the animal model experiment. Both B. holmesii isolates were not cytotoxic for HTE cell lines, even after eight hours of contact. We also assessed their invasive properties and persistence within HTE cells. FR4020 was more invasive than the other $B$. holmesii isolates tested (Figure 2), but all these isolates were significantly less invasive than a recently collected $B$. pertussis isolate $(p<$ 0.05 ). Moreover, they did not persist or multiply in epithelial cells over a period of 48 hours.

\section{Discussion}

We compared an isolate from a man with sickle cell anemia and B. holmesii bacteremia (Bho1, [23]) with another isolated from the respiratory tract of a woman with

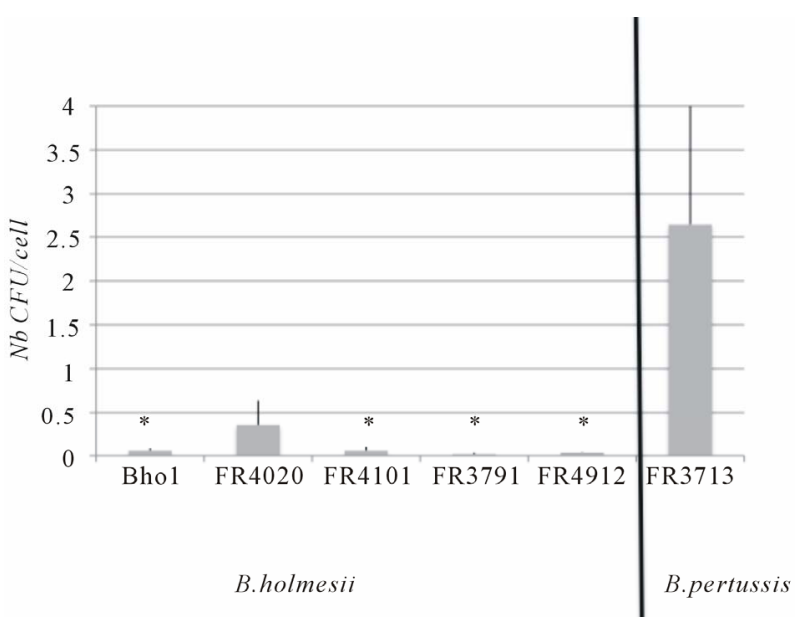

Each isolate was added to an individual well of a 24-well tissue culture plate containing $5 \times 10^{5}$ cells/ml, to achieve a ratio of 100 bacteria per cell. The invasion of HTE cells was assayed as described by Bouchez 2009 [26]. The results shown are the means and standard deviations of at least three experiments. The symbol $\left(^{*}\right)$ indicates $p<0.05$ in comparison with the $B$. pertussis strain FR3713.

Figure 2. Invasive properties of $B$. holmesii isolates.

whooping cough syndrome (FR4020, [6]).

These two isolates had PFGE profiles identical to each other and to those of isolates from the Institut Pasteur and National Reference Center collections [6,20,23], regardless of the origin or year of collection of these strains. None of the toxins and adhesins involved in the pathogenicity of classical Bordetella species, were detected in suspensions of the isolates with specific antibodies raised against purified $B$. pertussis virulence factors, confirming our previous findings $[20,23]$. Thus, these proteins are either not produced by $B$. holmesii isolates or the antibodies raised against the classical Bordetella virulence 
factors do not cross-react with the corresponding proteins in B. holmesii. Transcriptomic analyses were not performed since most of genes encoding virulence factors are not harbored in the genome of the $B$. holmesii isolates.

The bvgAS two-component regulatory system controls the expression of most virulence factors in Bordetella species. Regulatory sensors and activators have already been described in B. holmesii, with the identification of proteins with sequences 59.9\% identical to that of BvgS from B. pertussis and $72.2 \%$ identical to that of BvgA from $B$. pertussis [4]. Gerlach and coworkers [4] demonstrated that the cytoplasmic transduction domain of the BvgS histidine kinases of $B$. pertussis and B. holmesii were functionally interchangeable, whereas the extracellular sensor domains of these two proteins did not seem to respond to the same signals. We found that two of the isolates tested (Bho1 and CIP104394) had a truncated, and therefore nonfunction BvgA protein, because of the insertion of an A residue into $b v g A$, as previously described [3]. However, all the other B. holmesii isolates (including FR4020) had identical bvgS and bvgA sequences, whatever their origin (blood or respiratory), suggesting that regulation via the bvg two-component system should be similar in isolates from blood and respiratory samples.

Link and coworkers [5] described an FHA-related protein in $B$. holmesii. We confirmed the presence of such an fhaB-bho gene in Bho1 and FR4020. All the isolates collected after 2009 presented the same sequence, with a single non-silent SNP in the signal domain of the protein. Upstream from fhaB-bho, Link et al. [5] also demonstrated the presence of four heptanucleotide invertedrepeat sequences. This motif is very similar to the BvgA binding site in the $B$. pertussis fhaB promoter region, highlighting a possible role for $B$. holmesii BvgA in regulating the transcription of fhaB-bho. We found no genetic variation in this particular region for the $B$. holmesii isolates tested. Despite the presence of the gene, FhaB-bho was not detected by western blotting on suspensions of the isolates tested with specific antibodies raised against purified $B$. pertussis FHA. This is not surprising, because the amino-acid sequence of FhaB-bho is only $39 \%$ identical to that of $B$. pertussis FHA. Furthermore, the amino-acid sequence of FhaB-bho is 55\% identical to that of a similar protein present in B. avium [5,31], and we were unable to detect this protein by probing $B$. avium extracts with the same antibodies raised against purified B. pertussis FHA.

A locus analogous to fimABCD-fhaC was detected in the genomes of both Bho1 and FR4020. Such a locus has also been documented in classical Bordetella and in $B$. avium, in which it has been shown to be functional, regulated by temperature and involved in adhesion to the host respiratory tract tissues [32]. However, we detected no fimbrial proteins in the $B$. holmesii suspensions tested, with antibodies specific for the $B$. pertussis fimbrial proteins Fim 2 and Fim 3.

A CDS analogous to ampG was observed in both isolates. AmpG is an integral cytoplasmic membrane protein involved in peptidoglycan fragment recycling [33]. In $B$. pertussis, ampG gene has an insertion sequence IS481 that may affect its transcription, leading to the release of great quantities of Tracheal CytoToxin (TCT) a disaccharide-tetrapeptide monomer of peptidoglycan in culture supernantants [30,34]. No such insertion sequence is found around $a m p G$ sequence in $B$. holmesii suggesting that the level of TCT released should not be as high as for $B$. pertussis but rather lower as observed for other Bordetella species.

Diavatopoulos et al. [2] characterized a putative pathogenic island in the genome of $B$. holmesii isolates containing a functional iron-regulated alcaligin operon (known as the iron uptake island, or IUI) and other genes. This pathogenic island may have been acquired from $B$. pertussis, in which it is involved in the biosynthesis, export and uptake of the alcaligin siderophore in the absence of iron, leading to iron starvation. In microarray studies, Mooi et al. [11] reported the presence of this alcaligin operon in the genome of $B$. holmesii isolated from Dutch patients with pertussis-like illness. This operon (alcABCDERS-fauA) is conserved in the genome of Bho1 and FR4020, and its sequence displays only minor differences with respect to the sequences reported for $B$. pertussis BP2456 to BP2463. The IUI [2] also contains five genes (bhoABCDE) thought to be specific to $B$. holmesii species. We confirm their presence in Bho1 and FR4020. Furthermore, the sequences of these genes were found to be identical to the sequences submitted to GenBack (DQ402419) in both isolates, regardless of their origin (blood and a respiratory sample).

We identified two loci involved in aromatic compound metabolism. One of these loci contains genes encoding a benzene monooxygenase ( $b m o A)$ and a toluene O-xylene monooxygenase subunit similar to that of Pseudomonas mendocina, a Gram-negative environmental bacterium that can cause opportunistic nosocomial infections, such as infective endocarditis and spondylodiscitis [35]. The other locus contains genes displaying sequence identity to hypothetical protein-encoding genes from Azoarcus aromaticum, a proteobacterium capable of breaking down organic pollutants. The putative acquisition of proteins involved in aromatic compound metabolism has been reported for $B$. petrii, the only Bordetella species for which an environmental stage has been demonstrated [36,37].

Our genomic analysis did not distinguish between isolates responsible for bacteremia and those responsible 
for respiratory disease. These findings were supported by the phylogenetic analysis based on partial sequences from $b v g A / b v g S / f h a B$ and fimC. Isolates segregated according to the time at which they were collected rather than the type of source (blood or respiratory samples).

We already know that the genome of $B$. holmesii harbors IS481 sequences, but with fewer copies than are present in the genome of $B$. pertussis. This is why $B$. holmesii infection is diagnosed on the basis of a real-time PCR targeting a segment of the housekeeping gene recA (GenBank accession AF399664), which is polymorphic between B. holmesii, B. pertussis, B. parapertussis, $B$. bronchiseptica, B. hinzii, and B. avium [7-9]. This sequence was found to be fully conserved in both isolates, Bho1 and FR4020. Mooi et al. [11] recently used bhoE to confirm the identification of $B$. holmesii isolates based on 16s rRNA sequencing. Bho1 and FR4020 present a bhoE sequence identical to that in GenBank (DQ402419). $O m p A$, encoding a porin-like protein, is sometimes preferred for identification purposes, because it displays a higher degree of mutational variation than 16S rRNA [12]. The sequences of ompA in Bho1 and FR4020 were $100 \%$ identical to that in GenBank for other B. holmesii isolates (Accession no.: AM748266, AM748567 and JQ650246). Due to the high degree of similarity between the targeted sequences and the low level of divergence between $B$. holmesii isolates, current diagnostic tools cannot differentiate between isolates recovered from blood and those recovered from respiratory samples.

We found that $B$. holmesii isolates were not cytotoxic to the cells tested and that none of these isolates was lethal in a murine model of infection. These results are not surprising because no orthologs of either AC-Hly, which is responsible for macrophage cell death through apoptosis [28], or BteA, which is responsible for epithelial cell death through necrosis [38], have been found. No other cytotoxic agent seems to be produced by $\mathrm{B}$. holmesii. Furthermore, although FR4020 was slightly more invasive than the other isolates of $B$. holmesii tested, none of these isolates was particular invasive. We observed no difference in invasive properties for the Bho1 isolate, which has a nonfunctional BvgA protein, suggesting that Bvg system is unlikely to control the proteins involved in cell invasion processes.

In classical Bordetella species, adhesins and toxins are key factors in the interactions between bacteria and cells [39]. No ortholog of pertactin has been identified in $B$. holmesii, but the fhaB-bho gene of this species has some similarities with to that of the $B$. pertussis fhaB gene encoding the adhesin FHA. FHA promotes the invasion of human respiratory epithelial cells through the interaction of its RGD motif with the host cell 5-1 integrin [40]. B. holmesii produces an FHA-related protein, but this protein more closely resembles that of B. avium and contains a KGD motif rather than an RGD motif [5]. The role of this KGD motif in interactions with cells has yet to be established. Finally, a fimbrial locus has been reported for B. holmesii isolates, as for B. avium, in which it encodes fimbrial proteins involved in adhesion to the host respiratory epithelium [5]. The slightly greater invasive capacity of FR4020 cannot be linked to sequence differences in either fhaB-bho or fimC, because FR3791, an isolate harboring the same sequences, is as poorly invasive as the other $B$. holmesii isolates tested. Other proteins may thus be involved. None of the isolates tested persisted or multiplied in HTE cells over a period of 48 hours, regardless of their origin.

\section{Conclusions}

We were unable to identify any particular traits specific to $B$. holmesii isolates collected from blood or to isolates causing pertussis-like symptoms. We nevertheless report that Bho1 is unusual in having an incomplete BvgA protein. The more recent isolates tested (i.e. collected after 2009) had identical $b v g S, b v g A$ and fhaB sequences, regardless of their origin. We identified a fimbrial locus and genes involved in aromatic compound metabolism in both Bho1 and FR4020. Our genomic comparisons were restricted to particular genes, due to the absence of a reference genome for $B$. holmesii. A whole-genome annotation for a recently collected B. holmesii isolate would facilitate future genomic studies. Using in vitro cellular and in vivo animal models, we were unable to differentiate between isolates from blood and isolates from respiratory samples: none of these isolates was cytotoxic to J774.A1 murine macrophage cells or HTE cells. In addition, all of the isolates tested were only weakly invasive and none multiplied or persisted in HTE cells over a 48 hour period. Many questions about $B$. holmesii remain unanswered:

- Is B. holmesii a human respiratory pathogen or an opportunistic bacterium carried mostly by adolescents and adults (and, in rare cases, by newborns) but only detected during an episode of coughing due to another Bordetella species (as suggested by Rodgers et al., [10]) or another microorganism, such as a virus? Systematic searches for other respiratory pathogens must be undertaken. For example, in most studies, $B$. holmesii was isolated from respiratory samples from patients with pertussis-like symptoms, but tests for $B$. pertussis or $B$. parapertussis infections or viral infections were not systematically carried out.

- Can B. holmesii be carried asymptomatically? It is recovered from the blood of immunocompromised patients, such as asplenic patients and patients on chemotherapy [19]. It is probably important to obtain a nasopharyngeal swab from all patients with $B$. holmesii bacteremia. 
- Is B. holmesii adapting to humans? Multilocus sequence typing analysis revealed that $B$. holmesii is not a classical Bordetella species, instead lying at a similar distance from this group to B. avium [2]. Weyant et al. [1], in their first description of this species, reported that $B$. holmesii isolates had a cellular fattyacid profile closer to that of $B$. avium than to that of classical Bordetella species. Our genomic comparisons, based on only a few genes (as bvg fhaB, fim), also tended to suggest that the $B$. holmesii genome was closer to that of $B$. avium, which is thought to be a strictly avian pathogen. Nevertheless, human cases of respiratory disease associated with $B$. avium have already been reported in patients with cystic fibrosis [41], and B. avium isolates have also been collected from patients with pneumonia, thereby demonstrating that B. avium can also act as an opportunistic pathogen in humans, despite having an avian reservoir [42]. However, $B$. holmesii has acquired some genes from $B$. pertussis. It is thus possible that these two species coexist and exchange genetic material. Yih [17] and Rodgers [10] reported the cocirculation of B. pertussis and $B$. holmesii during outbreaks of pertussis. Is there a link between B. avium and B. holmesii? Does $B$. holmesii have an extrahuman reservoir that has yet to be identified? The sequencing and annotation of a complete $B$. holmesii genome are required, together with detailed genomic comparisons, to address these questions.

- Can B. holmesii be transmitted between humans? During an outbreak in Japan, Kamiya [13] observed epidemiological links between patients, suggesting that $B$. holmesii might have been transmitted from person to person. However, as previously stated, no other diagnostic tests were undertaken to check that $B$. holmesii was really the source of the whooping cough symptoms. This highlights the need to collect as much information about patients as possible during studies. It also highlights the possible transmission and carriage of $B$. holmesii between humans.

- Do vaccines against pertussis protect against $B$. holmesii? Zhang et al. [9] have already reported that neither whole-cell (wP) nor acellular (aP) B. pertussis vaccines confer protection against $B$. holmesii isolates in mice. Rodgers et al. [10] showed that aP booster vaccination was more effective against $B$. pertussis than against $B$. holmesii.

Clearly, further characterization of this bacterium is required. Much remains to be done and attentive monitoring should continue.

\section{Acknowledgements}

We thank the Collection of the Pasteur Institute for providing CIP isolates.
This work was supported by the Institut Pasteur Foundation, CNRS URA 3012, and GlaxoSmithKline Biologicals, Rixensart, Belgium.

\section{REFERENCES}

[1] R. S. Weyant, D. G. Hollis, R. E. Weaver, M. F. M. Amin, A. G. Steigerwalt, S. P. O’Connor, A. M. Whitney, M. I. Daneshvar, C. W. Moss and D. J. Brenner, "Bordetella holmesii sp. nov., a New Gram-Negative Species Associated with Septicemia,” Journal of Clinical Microbiology, Vol. 33, No. 1, 1995, pp. 1-7.

[2] D. A. Diavatopoulos, C. A. Cummings, H. G. van der Heide, M. van Gent, S. Liew, D. A. Relman and F. R. Mooi, "Characterization of a Highly Conserved Island in the Otherwise Divergent Bordetella holmesii and Bordetella pertussis Genomes,” Journal of bacteriology, Vol. 188, No. 24, 2006, pp. 8385-8394. doi:10.1128/JB.01081-06

[3] G. Gerlach, S. Janzen, D. Beier and R. Gross, "Functional Characterization of the bvgAS Two-Component System of Bordetella holmesii," Microbiology, Vol. 150, No. 11, 2004, pp. 3715-3729. doi:10.1099/mic.0.27432-0

[4] A. Horvat and R. Gross, "Molecular Characterization of the BvgA Response Regulator of Bordetella holmesii," Microbiology Research, Vol. 164, No. 3, 2009, pp. 243252. doi:10.1016/j.micres.2006.11.015

[5] S. Link, K. Schmitt, D. Beier and R. Gross, "Identification and regulation of expression of a gene encoding a filamentous hemagglutinin-related protein in Bordetella holmesii,” BMC Microbiology, Vol. 7, No. 2007, p. 100.

[6] A. Tizolova, N. Guiso and S. Guillot, "Insertion Sequences Shared by Bordetella Species and Implications for the Biological Diagnosis of Pertussis Syndrome," European Journal of Clinical Microbiology Infectious Diseases, Vol. 32, No. 1, 2013, pp. 89-96. doi:10.1007/s10096-012-1718-3

[7] O. Vielemeyer, J. Y. Crouch, S. C. Edberg and J. G. Howe, "Identification of Bordetella pertussis in a Critically Ill Human Immunodeficiency Virus-Infected Patient by Direct Genotypical Analysis of Gram-Stained Material and Discrimination from $B$. holmesii by Using a Unique recA Gene Restriction Enzyme Site,” Journal of Clinical Microbiology, Vol. 42, No. 2, 2004, pp. 847-849. doi:10.1128/JCM.42.2.847-849.2004

[8] M. Antila, Q. He, C. de Jong, I. Aarts, H. Verbakel, S. Bruisten, S. Keller, M. Haanpera, J. Makinen, E. Eerola, M. K. Viljanen, J. Mertsola and A. van der Zee, “Bordetella holmesii DNA Is Not Detected in Nasopharyngeal Swabs from Finnish and Dutch Patients with Suspected Pertussis,” Journal of Medical Microbiology, Vol. 55, No. 8, 2006, pp. 1043-1051. doi:10.1099/jmm.0.46331-0

[9] J. L. Guthrie, A. V. Robertson, P. Tang, F. Jamieson and S. J. Drews, "Novel Duplex Real-Time PCR Assay Detects Bordetella holmesii in Specimens from Patients with Pertussis-Like Symptoms in Ontario, Canada,” Journal of Clinical Microbiology, Vol. 48, No. 4, 2010, pp. 14351437. doi:10.1128/JCM.02417-09

[10] L. Rodgers, S. W. Martin, A. Cohn, J. Budd, M. Marcon, 
A. Terranella, S. Mandal, D. Salamon, A. Leber, M. L. Tondella, K. Tatti, K. Spicer, A. Emanuel, E. Koch, L. McGlone, L. Pawloski, M. Lemaile-Williams, N. Tucker, R. Iyer, T. A. Clark and M. Diorio, "Epidemiologic and Laboratory Features of a Large Outbreak of PertussisLike Illnesses Associated with Co-Circulating Bordetella holmesii and Bordetella pertussis-Ohio, 2010-2011," Clinical Infectious Diseases, Vol. 56, No. 3, 2013, pp. 322331. doi:10.1093/cid/cis888

[11] F. R. Mooi, S. Bruisten, I. Linde, F. Reubsaet, K. Heuvelman, S. van der Lee and J. K. A, "Characterization of Bordetella holmesii Isolates from Patients with PertussisLike Illness in the Netherlands," FEMS Immunology and Medical Microbiology, Vol. 64, No. 2, 2012, pp. 289-291. doi:10.1111/j.1574-695X.2011.00911.X

[12] S. Jonckheere, T. De Baere, P. Schroeyers, O. Soetens, A. De Bel and I. Surmont, "Prosthetic Valve Endocarditis Caused by Bordetella holmesii, an Acinetobacter LookAlike,” Journal of Medical Microbiology, Vol. 61, No. 6, 2012, pp. 874-877. doi:10.1099/jmm.0.038695-0

[13] H. Kamiya, N. Otsuka, Y. Ando, F. Odaira, S. Yoshino, K. Kawano, H. Takahashi, T. Nishida, Y. Hidaka, H. Toyoizumi-Ajisaka, K. Shibayama, K. Kamachi, T. Sunagawa, K. Taniguchi and N. Okabe, "Transmission of Bordetella holmesii during Pertussis Outbreak, Japan,” Emerging Infectious Diseases, Vol. 18, No. 7, 2012, pp. 1166-1169. doi:10.3201/eid1807.120130

[14] D. M. Livovsky, D. Leibowitz, C. Hidalgo-Grass, V. Temper, S. Salameh and M. Korem, "Bordetella holmesii Meningitis in an Asplenic Patient with Systemic Lupus Erythematosus,” Journal of Medical Microbiology, Vol. 61, No. 8, 2012, pp. 1165-1167. doi:10.1099/jmm.0.038208-0

[15] E. Mazengia, E. A. Silva, J. A. Peppe, R. Timperi and H. George, "Recovery of Bordetella holmesii from Patients with Pertussis-Like Symptoms: Use of Pulsed-Field Gel Electrophoresis to Characterize Circulating Strains,” Journal of Clinical Microbiology, Vol. 38, No. 6, 2000, pp. 2330-2333.

[16] E. Njamkepo, S. Bonacorsi, M. Debruyne, S. A. Gibaud, S. Guillot and N. Guiso, "Significant Finding of Bordetella holmesii DNA in Nasopharyngeal Samples from French Patients with Suspected Pertussis,” Journal of Clinical Microbiology, Vol. 49, No. 12, 2011, pp. 43474348. doi:10.1128/JCM.01272-11

[17] W. K. Yih, E. A. Silva, J. Ida, N. Harrington, S. M. Lett and H. George, "Bordetella holmesii-Like Organisms Isolated from Massachussetts Patients with Pertussis-Like Symptoms,” Emerging Infectious Diseases, Vol. 5, No. 3, 1999, pp. 441-443. doi:10.3201/eid0503.990317

[18] X. Zhang, L. S. Weyrich, J. S. Lavine, A. T. Karanikas and E. T. Harvill, "Lack of Cross-Protection against Bordetella holmesii after Pertussis Vaccination," Emerging Infectious Diseases, Vol. 18, No. 11, 2012, pp. 17711779.

[19] T. Chambaraud, Z. Dickson, G. Ensergueix, O. Barraud, M. Essig, C. Lacour, J. Allard, F. Bocquentin, J. C. Aldigier and J. P. Rerolle, "Bordetella holmesii Bacteremia in a Renal Transplant Recipient: Emergence of a New Pathogen,” Transplant Infectious Disease, Vol. 14, No. 6,

\section{2, pp. E134-E136. doi:10.1111/tid.12009}

[20] M. I. Panagopoulos, M. Saint Jean, D. Brun, N. Guiso, S. Bekal, P. Ovetchkine and B. Tapiero, "Bordetella holmesii Bacteremia in Asplenic Children: Report of Four Cases Initially Misidentified as Acinetobacter lwoffii,” Journal of Clinical Microbiology, Vol. 48, No. 10, 2010, pp. 37623764. doi:10.1128/JCM.00595-10

[21] D. Bottero, M. M. Griffith, C. Lara, D. Flores, L. Pianciola, M. E. Gaillard, M. Mazzeo, M. I. Zamboni, M. J. Spoleti, E. Anchart, D. Ruggeri, C. Sorhouet, S. Fiori, M. Galas, M. L. Tondella and D. F. Hozbor, "Bordetella holmesii in Children Suspected of Pertussis in Argentina,” Epidemiology and infection, Vol. 141, No. 4, 2013, pp. 714-717. doi:10.1017/S095026881200132X

[22] C. Miranda, L. Porte and P. Garcia, "Bordetella holmesii in Nasopharyngeal Samples from Chilean Patients with Suspected Bordetella pertussis Infection,” Journal of Clinical Microbiology, Vol. 50, No. 4, 2012, p. 1505; Author Reply 1506.

[23] E. Njamkepo, F. Delisle, I. Hagege, G. Gerbaud and N. Guiso, "Bordetella holmesii Isolated from a Patient with Sickle Cell Anemia: Analysis and Comparison with Other Bordetella holmesii Isolates,” Clinical Microbiology and Infection, Vol. 6, No. 3, 2000, pp. 131-136. doi:10.1046/j.1469-0691.2000.00032.x

[24] V. Caro, E. Njamkepo, S. C. Van Amersfoorth, F. R. Mooi, A. Advani, H. O. Hallander, Q. He, J. Mertsola, M. Riffelmann, C. Vahrenholz, C. H. Von Konig and N. Guiso, "Pulsed-Field Gel Electrophoresis Analysis of Bordetella pertussis Populations in Various European Countries with Different Vaccine Policies," Microbes Infection, Vol. 7, No. 7-8, 2005, pp. 976-982. doi:10.1016/j.micinf.2005.04.005

[25] C. Weber, C. Boursaux-Eude, G. Coralie, V. Caro and N. Guiso, "Polymorphism of Bordetella pertussis Isolates Circulating the Last Ten Years in France, Where a Single Effective Whole-Cell Vaccine Has Been Used for More Than Thirty Years,” Journal of Clinical Microbiology, Vol. 39, No. 12, 2001, pp. 4396-4403. doi:10.1128/JCM.39.12.4396-4403.2001

[26] V. Bouchez, D. Brun, T. Cantinelli, G. Dore, E. Njamkepo and N. Guiso, "First Report and Detailed Characterization of B. pertussis Isolates Not Expressing Pertussis Toxin or Pertactin,” Vaccine, Vol. 27, No. 43, 2009, pp. 6034-6041. doi:10.1016/j.vaccine.2009.07.074

[27] P. Software, "Phylogeny Inference Package (PHYLIP),” In: J. Felsenstein, Ed., PHYLIP-Phylogeny Inference Package (Version 3.2), Cladistics 5, Department of Genome Sciences, University of Washington, Seattle, 1989, pp. 164166.

[28] N. Khelef and N. Guiso, "Induction of Macrophage Apoptosis by Bordetella pertussis Adenylate Cyclase-Hemolysin,” FEMS Microbiology Letters, Vol. 134, No. 1, 1995, pp. 27-32. doi:10.1111/j.1574-6968.1995.tb07909.x

[29] P. Gueirard, L. Bassinet, I. Bonne, M. C. Prevost and N. Guiso, "Ultrastructural Analysis of the Interactions between Bordetella pertussis, Bordetella parapertussis and Bordetella bronchiseptica and Human Tracheal Epithelial Cells,” Microbial pathogenesis, Vol. 38, No. 1, 2005, pp. 
41-46. doi:10.1016/j.micpath.2004.08.003

[30] S. Mattoo, A. K. Foreman-Wykert, P. A. Cotter and J. F. Miller, "Mechanisms of Bordetella Pathogenesis," Frontiers in Bioscience, Vol. 6, No. 86, 2001, p. 1.

[31] M. Sebaihia, A. Preston, D. J. Maskell, H. Kuzmiak, T. D. Connell, N. D. King, P. E. Orndorff, D. M. Miyamoto, N. R. Thomson, D. Harris, A. Goble, A. Lord, L. Murphy, M. A. Quail, S. Rutter, R. Squares, S. Squares, J. Woodward, J. Parkhill and L. M. Temple, "Comparison of the Genome Sequence of the Poultry Pathogen Bordetella avium with Those of B. bronchiseptica, B. pertussis, and B. parapertussis Reveals Extensive Diversity in Surface Structures Associated with Host Interaction,” Journal of Bacteriology, Vol. 188, No. 16, 2006, pp. 6002-6015. doi:10.1128/JB.01927-05

[32] S. B. Loker, L. M. Temple and A. Preston, "The Bordetella avium BAV1965-1962 Fimbrial Locus Is Regulated by Temperature and Produces Fimbriae Involved in Adherence to Turkey Tracheal Tissue," Infection and Immunity, Vol. 79, No. 6, 2011, pp. 2423-2429. doi:10.1128/IAI.01169-10

[33] A. Chahboune, M. Decaffmeyer, R. Brasseur and B. Joris, "Membrane Topology of the Escherichia coli AmpG Permease Required for Recycling of Cell Wall Anhydromuropeptides and AmpC Beta-Lactamase Induction,” Antimicrobial agents and chemotherapy, Vol. 49, No. 3, 2005, pp. 1145-1149. doi:10.1128/AAC.49.3.1145-1149.2005

[34] W. E. Goldman and B. T. Cookson, "Structure and Functions of the Bordetella Tracheal Cytotoxin," The Tokai Journal of Experimental and Clinical Medicine, Vol. 13, 1988, pp. 187-191.

[35] K. M. Yen, M. R. Karl, L. M. Blatt, M. J. Simon, R. B. Winter, P. R. Fausset, H. S. Lu, A. A. Harcourt and K. K. Chen, "Cloning and Characterization of a Pseudomonas mendocina KR1 Gene Cluster Encoding Toluene-4-Monooxygenase,” Journal of Bacteriology, Vol. 173, No. 17, 1991, pp. 5315-5327.

[36] R. Gross, C. A. Guzman, M. Sebaihia, V. A. dos Santos, D. H. Pieper, R. Koebnik, M. Lechner, D. Bartels, J.
Buhrmester, J. V. Choudhuri, T. Ebensen, L. Gaigalat, S. Herrmann, A. N. Khachane, C. Larisch, S. Link, B. Linke, F. Meyer, S. Mormann, D. Nakunst, C. Ruckert, S. Schneiker-Bekel, K. Schulze, F. J. Vorholter, T. Yevsa, J. T. Engle, W. E. Goldman, A. Puhler, U. B. Gobel, A. Goesmann, H. Blocker, O. Kaiser and R. Martinez-Arias, "The Missing Link: Bordetella petrii Is Endowed with Both the Metabolic Versatility of Environmental Bacteria and Virulence Traits of Pathogenic Bordetellae," BMC Genomics, Vol. 9, 2008, p. 449.

[37] M. Lechner, K. Schmitt, S. Bauer, D. Hot, C. Hubans, E. Levillain, C. Locht, Y. Lemoine and R. Gross, "Genomic Island Excisions in Bordetella petrii,” BMC Microbiology, Vol. 9, 2009, p. 141.

[38] K. E. Stockbauer, A. K. Foreman-Wykert and J. F. Miller, "Bordetella Type III Secretion Induces Caspase 1-Independent Necrosis,” Cell Microbiology, Vol. 5, No. 2, 2003, pp. 123-132. doi:10.1046/j.1462-5822.2003.00260.x

[39] L. Bassinet, P. Gueirard, B. Maitre, B. Housset, P. Gounon and N. Guiso, "Role of Adhesins and Toxins in Invasion of Human Tracheal Epithelial Cells by Bordetella pertussis," Infection and Immunity, Vol. 68, No. 4, 2000, pp. 1934-1941. doi:10.1128/IAI.68.4.1934-1941.2000

[40] Y. Ishibashi, D. A. Relman and A. Nishikawa, "Invasion of Human Respiratory Epithelial Cells by Bordetella pertussis: Possible Role for a Filamentous Hemagglutinin Arg-Gly-Asp Sequence and Alpha5beta1 Integrin," Microbial Pathogenesis, Vol. 30, No. 5, 2001, pp. 279-288. doi:10.1006/mpat.2001.0432

[41] T. Spilker, A. A. Liwienski and J. J. LiPuma, "Identification of Bordetella spp. in Respiratory Specimens from Individuals with Cystic Fibrosis," Clinical Microbiology Infection, Vol. 14, No. 5, 2008, pp. 504-506. doi:10.1111/j.1469-0691.2008.01968.x

[42] A. T. Harrington, J. A. Castellanos, T. M. Ziedalski, J. E. Clarridge 3rd and B. T. Cookson, "Isolation of Bordetella avium and Novel Bordetella Strain from Patients with Respiratory Disease,” Emerging Infectious Diseases, Vol. 15, No. 1, 2009, pp. 72-74. doi:10.3201/eid1501.0716 